\author{
Jacques Laskar \\ Service de Mécanique Céleste du Bureau des Longitudes \\ Equipe de Recherche Associée au CNRS \\ 77 Avenue Denfert-Rochereau F-75014 Paris, France. \\ Jet Propulsion Laboratory \\ California Institute of Technology \\ 4800 Oak Grove Drive, Pasadena, California, 91109 USA
}

\begin{abstract}
The relativistic and lunar perturbations must be included in a realistic theory of the secular evolution of the planetary elements. In our general theory, we include the first order of these perturbations. Comparison with more elaborated studies shows that it is sufficient with respect to the accuracy of our theory.
\end{abstract}

\title{
INTRODUCTION
}

The long period variations of the orbital elements of the solar system are of increasing interest since the development of the Milankovitch theory of climate (Berger et al., 1984). The early solution of Brouwer and Van Woerkom (1950) contains the first order linear terms and the second order terms in the Jupiter-Saturn couple computed by Hill (1897) up to degree 5 in the eccentricity. Bretagnon (1974) took into account all the second order terms of degree 3 in eccentricity and inclination for the 8 major planets.

We have undertaken a new general theory for the 8 planets, based on the works of Brumberg and Egorova (1971), Brumberg (1980), Chapront (1970), Abu El Ata and Chapront (1975), and on Duriez's theory of the 4 outer planets $(1977,1979)$. Special attention is given to estimating the accuracy of the solution, which includes all the terms of order 2 up to degree 5 in eccentricity and inclination. The relativistic and lunar perturbations must be included in a realistic solution (Bretagnon, 1984a). * This paper was not formally presented during the symposium because the
author was unable to attend due to visa problems.

85

J. Kovalevsky and V. A. Brumberg (eds.), Relativity in Celestial Mechanics and Astrometry, 85-92.

(C) 1986 by the IAU. 


\section{THE SECULAR EQUATIONS}

The elliptic elements are denoted by the classical notations $a, e, i, \Omega, \varpi, \varepsilon$. We use also the variables $(p, q, z, \varsigma, \mathbf{k}, \mathbf{h}, \mathbf{q}, \mathbf{p})$ defined by:

$$
\begin{aligned}
& a=A(1+p)^{-2 / 3} \Longleftrightarrow n=N(1+p) \\
& \lambda=N t-\sqrt{-1} q=\int n d t+\varepsilon \\
& z=e \exp \sqrt{-1} \varpi=\mathbf{k}+\sqrt{-1 \mathrm{~h}} \\
& \zeta=\sin \frac{i}{2} \exp \sqrt{-1} \Omega=q+\sqrt{-1} \mathrm{p}
\end{aligned}
$$

where $n$ is the mean motion, $N$ the observed mean mean motion, and $A$ the semi-major axis of reference related to $N$ by the Kepler relation $N^{2} A^{3}=n^{2} a=G M_{\odot}\left(1+m / M_{\odot}\right)$ where $G$ is the gravitation constant, $M_{\odot}$ the solar mass, and $m$ the planetary mass.

The variations of the osculating elements $(p, q, z, \zeta)$ are given by the classical Lagrange equations :

$$
\begin{aligned}
& \frac{d p}{d t}=\frac{-3 \sqrt{-1}}{N A^{2}}(1+p)^{4 / 3} \frac{\partial R}{\partial p} \\
& \frac{d q}{d t}=\sqrt{-1} N p+\frac{\sqrt{-1}(1+p)^{1 / 3}}{N A^{2}}\left[3(1+p) \frac{\partial R}{\partial p}+\phi \psi z\left(\frac{\partial R}{\partial z}+\bar{z} \frac{\partial R}{\partial \bar{z}}\right)+\frac{1}{2 \phi}\left(\zeta \frac{\partial R}{\partial \zeta}+\bar{\zeta} \frac{\partial R}{\partial \bar{\zeta}}\right)\right] \\
& \frac{d z}{d t}=\frac{\sqrt{-1}(1+p)^{1 / 3}}{N A^{2}}\left[2 \psi \frac{\partial R}{\partial \bar{z}}-\phi \psi z \frac{\partial R}{\partial q}+\frac{z}{2 \phi}\left(\zeta \frac{\partial R}{\partial \zeta}+\bar{\zeta} \frac{\partial R}{\partial \bar{\zeta}}\right)\right] \\
& \frac{d \zeta}{d t}=\frac{\sqrt{-1}(1+p)^{1 / 3}}{2 \phi N A^{2}}\left[\frac{\partial R}{\partial \bar{\zeta}}-\varsigma \frac{\partial R}{\partial q}+\varsigma\left(-z \frac{\partial R}{\partial z}+\bar{z} \frac{\partial R}{\partial \bar{z}}\right)\right]
\end{aligned}
$$

where $\phi=\sqrt{1-z \bar{z}}$ and $\psi=1 /(1+\phi)$. The disturbing function $R$ is expanded in Poisson series depending on the variables $p_{i}, q_{i}, z_{i}, \bar{z}_{i}, \zeta_{i}, \bar{\zeta}_{i}$ and on the time $t$ (Duriez, 1977; Laskar, 1985a).

We gather the variables $p_{i}, q_{i}, z_{i}, \bar{z}_{i}, \zeta_{i}, \bar{\zeta}_{i}$ in a single vector $V$. The differential system (2) is then written in the short form:

$$
\frac{d V}{d t}=\Lambda(V, t)
$$

To integrate this system, we split $V$ in two parts:

$$
V=V_{0}+\Delta V\left(V_{0}, t\right)
$$

where $V_{0}$ denotes the secular part of the variables and $\Delta V$ the short period part, depending on $V_{0}$ and $t$. Substitution in (3) and identification by order gives then the secular differential system of order 2 :

$$
\frac{d V_{0}}{d t}=\left\langle\Lambda\left(V_{0}, t\right)\right\rangle+\left\langle\frac{\partial \Lambda}{\partial V_{0}}\left(V_{0}, t\right) \Delta_{1} V\left(V_{0}, t\right)\right\rangle
$$


where $\langle x\rangle$ is the average of $x$ over the time and $\Delta_{1} V$ is the short period solution of order 1 (Duriez, 1977, 1979; Laskar, 1985a). The secular system of order 1 is given by the first part of (5); it does not contain the contributions of the short period terms to the secular terms which arise from the products of the second part of (5).

We performed a extensive computation of the secular system (5) up to degree 5 in the eccentricity and inclination variables (Laskar, 1985a). In the equation (5), the time does not appear any more, nor the variables $q_{i}$ which are related to the longitudes. Besides, the secular part of $p_{i}$ are constant and they are replaced by their numerical values in (5). The secular system (5) depends then only on the variables $z_{i}, \bar{z}_{i}, \zeta_{i}, \bar{\zeta}_{i}$.

We denote by $\alpha$ the secular part of $\left(z_{1}, \ldots ., z_{8}, \varsigma_{1}, \ldots . \zeta_{8}\right)$ and isolate in (5) the differential system giving the variations of $\alpha$ :

$$
\frac{d \alpha}{d t}=\sqrt{-1}\left(\Phi_{1} \alpha+\Phi_{3}(\alpha, \bar{\alpha})+\Phi_{5}(\alpha, \bar{\alpha})\right)
$$

This system contains 153824 monomial terms. $\Phi_{1}$ is a real matrix with constant coefficients, $\Phi_{3}$ gathers the terms of degree 3 and $\Phi_{5}$ the terms of degree 5 .

\section{INTEGRATION OF THE EQUATIONS}

In order to avoid the problems of small divisors in the analytical integration of (6) (Laskar, 1984), we integrate (6) numerically integration which can be performed with a large step size of about 500 years. Moreover, we have also included in our differential system (6) the equations of (Kinoshita, 1977) for the secular evolution of precession and obliquity of the Earth which are then integrated together with the orbital elements.

A first numerical integration was made over 10000 years on each side of $\mathrm{J} 2000$. The solution is then expanded in Taylor series by numerical differentiation with respect to the time. It allow us to check the accuracy of the solution by direct comparisons with the analytical ephemeris VSOP82 (Bretagnon, 1982), and with the JPL numerical ephemeris DE102 (Newhall et al., 1983). Besides, the computation of the polynomial expansion of the secular terms up to high powers of the time has been used to extend VSOP82 over 6000 years for the construction of ephemerides aimed at historical computations (Laskar, 1985b; Bretagnon et al., 1985).

Over a longer span of time, the polynomial expansion in powers of the time is not useful. A representation of the solution as a quasi-periodic function should be preferred to give the main frequences of the solution. We have performed a numerical integration of the whole system (6) over 30 millions years which should be enough to derive a solution in a quasi-periodic form. This work is not finished yet and we shall present the results in a forthcoming paper.

\section{RELATIVISTIC AND LUNAR PERTURBATIONS}

In both numerical integrations, we have included some perturbations due to relativity and to the effect of the Moon. These perturbations are simplified models which contains 
only the first order terms. They do not consider the second order terms which could arise from the products of short period terms. We can check their accuracy by comparison with some more elaborated theories.

For relativity, we consider the first order terms in the motion of the perihelion (Brumberg, 1972; Lestrade and Bretagnon, 1982), limited to the post-newtonian approximation in $1 / c^{2}$. In the isotropic coordinates, it adds to (6) the term:

$$
\left.\frac{d z}{d t}\right|_{R}=\sqrt{-1} \frac{G M_{\odot}}{c^{2}} \times \frac{3 n}{a} \times \frac{1}{1-e^{2}} \times z
$$

The contribution of this term to the secular variations of the elliptical elements $z_{i}, \zeta_{i}$ are directly estimated by the comparison of the solution with and without the relativistic term (7). This contribution is given up to $t^{6}$ for the inner planets in Table 1 . For comparison, we put in Table 2 the complete secular terms for the same variables, derived from our theory and VSOP82 (Laskar, 1985b). In Table 1, we also put the term in $t$ and in $t^{2}$ computed by (Lestrade and Bretagnon, 1982). The constants they used were not exactly the same, and the coefficients of $t^{2}$ contains the second order terms coming from the products of newtonian and relativistic short period terms which do not exist in our solution. The discrepencies between the two solutions are very small and lead to differences smaller than $2 \times 10^{-7}$ after 10000 years in the eccentricities of Mercury and Mars, which is under our level of accuracy. Besides, the high powers of the relativistic contributions could be used to improve a newtonian solution.

The perturbation of the Moon is limited to a single constant term (Bretagon, 1984c):

$$
\left.\frac{d z_{3}}{d t}\right|_{L}=\sqrt{-1} \delta_{L} z_{3}
$$

where $\delta=3.192472 \times 10^{-7}$.

The same computations as with the relativity are made in this case and the results are presented in Table 3. The comparisons of the coefficients of $t$ and $t^{2}$ are made with the results of (Bretagon, 1984b) which computed the same quantities using the complete theory of the Moon ELP-2000/82 (Chapront-Touzé, Chapront, 1983), and his planetary theory VSOP82. His values includes the contribution of the short period terms in the secular terms of the Moon (the comparison cannot be made with the terms in $t^{3}$ of (Bretagon, 1984b) which are not complete). In this case also, the agreement is good, despite the very simple form of (8).

\section{CONCLUSION}

An accurate secular theory of the 8 planets must include the relativistic and lunar perturbations. Their first order expression given in (7) and (8) are sufficient with respect to the actual level of precision of our theory. An improved theory should probably include the second order terms from the relativity, but principally a more elaborated representation of the lunar perturbations on the Earth-Moon center of mass. 


\begin{tabular}{|c|c|c|c|c|}
\hline & $\mathbf{k} \times 10^{10}$ & $\mathrm{~h} \times 10^{10}$ & $q \times 10^{10}$ & $p \times 10^{10}$ \\
\hline \multicolumn{5}{|c|}{ MERCURY } \\
\hline$t$ & $\begin{array}{l}-41825896 \\
-41826163\end{array}$ & $\begin{array}{l}9306191 \\
9306388\end{array}$ & 0 & 0 \\
\hline$t^{2}$ & $\begin{array}{l}-2522666 \\
-2520879\end{array}$ & $\begin{array}{l}-11195643 \\
-11191877\end{array}$ & $\begin{array}{l}218161 \\
219308\end{array}$ & $\begin{array}{l}-362819 \\
-362065\end{array}$ \\
\hline $\begin{array}{l}t^{3} \\
t^{4} \\
t^{5} \\
t^{6}\end{array}$ & $\begin{array}{r}1497089 \\
47009 \\
-11559 \\
-3252\end{array}$ & $\begin{array}{r}-456098 \\
151595 \\
5896 \\
336\end{array}$ & $\begin{array}{r}129364 \\
-38383 \\
2841 \\
2467\end{array}$ & $\begin{array}{r}101391 \\
23255 \\
-9469 \\
1975\end{array}$ \\
\hline \multicolumn{5}{|c|}{ VENUS } \\
\hline$t$ & $\begin{array}{l}-211863 \\
-211875\end{array}$ & $\begin{array}{l}-187861 \\
-187857\end{array}$ & 0 & 0 \\
\hline$t^{2}$ & $\begin{array}{l}139204 \\
138041\end{array}$ & $\begin{array}{l}146492 \\
146104\end{array}$ & -7084 & 9087 \\
\hline $\begin{array}{l}t^{3} \\
t^{4}\end{array}$ & $\begin{array}{r}-83173 \\
-5071\end{array}$ & $\begin{array}{r}32988 \\
-22829\end{array}$ & $\begin{array}{r}-1085 \\
1625\end{array}$ & $\begin{array}{r}-2811 \\
769\end{array}$ \\
\hline$t^{5}$ & 5865 & 335 & -549 & 321 \\
\hline$t^{6}$ & 69 & 11 & -195 & -343 \\
\hline \multicolumn{5}{|c|}{ EARTH } \\
\hline$t$ & $\begin{array}{l}-303062 \\
-303066\end{array}$ & $\begin{array}{l}-69618 \\
-69617\end{array}$ & 0 & 0 \\
\hline$t^{2}$ & $\begin{array}{l}66348 \\
65664\end{array}$ & $\begin{array}{l}-133624 \\
-132712\end{array}$ & -947 & 2750 \\
\hline$t^{3}$ & 56321 & 24339 & -1485 & -607 \\
\hline$t^{4}$ & -6409 & 17256 & 76 & -639 \\
\hline$t^{5}$ & -4876 & -1706 & 110 & 150 \\
\hline$t^{6}$ & 446 & -445 & -10 & 124 \\
\hline \multicolumn{5}{|c|}{ MARS } \\
\hline$t$ & $\begin{array}{l}248226 \\
248226\end{array}$ & $\begin{array}{l}559105 \\
559103\end{array}$ & 0 & 0 \\
\hline$t^{2}$ & $\begin{array}{l}-448110 \\
-446324\end{array}$ & $\begin{array}{l}248285 \\
247322\end{array}$ & -516 & 2048 \\
\hline$t^{3}$ & -108734 & -184672 & -2558 & -399 \\
\hline$t^{4}$ & 50478 & -31382 & -159 & -2204 \\
\hline$t^{5}$ & 6041 & 9807 & 1474 & -283 \\
\hline$t^{6}$ & -1532 & 1222 & 331 & 736 \\
\hline
\end{tabular}

Table 1. Contribution of the relativity in the secular terms (Eq.7). The values in oldstyle are the corresponding values obtained by Lestrade and Bretagnon (1982), which include the second order terms due to the product of short period terms. The time $t$ is measured in units of 10000 julian years from J2000 (JD 2451545.0 ). 


\begin{tabular}{|c|c|c|c|c|}
\hline & $\mathbf{k} \times 10^{10}$ & $\mathrm{~h} \times 10^{10}$ & $q \times 10^{10}$ & $p \times 10^{10}$ \\
\hline \multicolumn{5}{|c|}{ MERCURY } \\
\hline & 2007233137 & 406156338 & 456355046 & \\
\hline$t$ & -552114624 & 143750118 & $\begin{array}{lll}65 & 433 & 117\end{array}$ & -127633657 \\
\hline$t^{2}$ & -18607467 & - 79744997 & -10713296 & -9134193 \\
\hline$t^{3}$ & 7904951 & -3043725 & 2245279 & 1898818 \\
\hline$t^{4}$ & 589540 & 811285 & -376780 & -640089 \\
\hline$t^{5}$ & -156482 & -78243 & -30978 & -25951 \\
\hline$t^{6}$ & - 52991 & 27580 & 10508 & 47156 \\
\hline \multicolumn{5}{|c|}{ VENUS } \\
\hline & -44928213 & 50668473 & 68241014 & 288228577 \\
\hline$t$ & 31259019 & - 36121239 & 138133826 & -40384791 \\
\hline$t^{2}$ & 6041681 & 18468752 & -10909716 & -62328916 \\
\hline$t^{3}$ & -6834889 & 328049 & - 18641793 & 2473042 \\
\hline$t^{4}$ & 493964 & -613650 & 601726 & 4228784 \\
\hline$t^{5}$ & 597550 & -168598 & 746057 & - 57042 \\
\hline$t^{6}$ & -109138 & -123616 & -40592 & -116943 \\
\hline \multicolumn{5}{|c|}{ EARTH } \\
\hline & -37408165 & 162844766 & 0 & 0 \\
\hline$t$ & - 82266699 & - 62030259 & -113469002 & 10180391 \\
\hline$t^{2}$ & 27626329 & - 33829810 & 12372674 & 47020439 \\
\hline$t^{3}$ & 11695572 & 8510121 & 12654170 & -5417367 \\
\hline$t^{4}$ & -2695722 & 2770542 & -1371808 & -2507948 \\
\hline$t^{5}$ & -715070 & -467407 & -320334 & 463486 \\
\hline$t^{6}$ & 218146 & -62395 & 5072 & 56431 \\
\hline \multicolumn{5}{|c|}{ MARS } \\
\hline & 853656025 & - 378997324 & 104704257 & 122844931 \\
\hline$t$ & $\begin{array}{lll}376 & 330 & 152\end{array}$ & 624657465 & 17138526 & -108020083 \\
\hline$t^{2}$ & - 246579527 & 155295878 & - 40776201 & -19221776 \\
\hline$t^{3}$ & -36760524 & - 63487894 & - 13883445 & 8718504 \\
\hline$t^{4}$ & 11112422 & - 6592895 & 916176 & 3090121 \\
\hline$t^{5}$ & 259071 & 729862 & 1759071 & 37687 \\
\hline$t^{6}$ & 7855 & 113707 & 112984 & 8722 \\
\hline
\end{tabular}

Table 2. Secular terms for the inner planets. The time $t$ is measured in units of 10000 julian years from J2000 (JD 2451 545.0). 


\begin{tabular}{|c|c|c|c|c|}
\hline & $\mathbf{k} \times 10^{10}$ & $\mathrm{~h} \times 10^{10}$ & $q \times 10^{10}$ & $p \times 10^{10}$ \\
\hline \multicolumn{5}{|c|}{ MERCURY } \\
\hline$t$ & 0 & 0 & 0 & 0 \\
\hline \multirow[t]{2}{*}{$t^{2}$} & -1710 & 5690 & 6 & 58 \\
\hline & -1712 & 5695 & & \\
\hline$t^{3}$ & -114 & -548 & -173 & -78 \\
\hline$t^{4}$ & 151 & -568 & -6 & -105 \\
\hline$t^{5}$ & -126 & -130 & 1 & 8 \\
\hline$t^{6}$ & -40 & -62 & 15 & 23 \\
\hline \multicolumn{5}{|c|}{ VENUS } \\
\hline$t$ & 0 & 0 & 0 & 0 \\
\hline$t^{2}$ & -18608 & 72095 & -233 & -174 \\
\hline & $-184^{80}$ & 71366 & & \\
\hline$t^{3}$ & -44274 & -13952 & 636 & 274 \\
\hline$t^{4}$ & 8931 & -10568 & -149 & 217 \\
\hline$t^{5}$ & 4594 & 109 & -329 & -203 \\
\hline$t^{6}$ & -1288 & -276 & 99 & -135 \\
\hline \multicolumn{5}{|c|}{ EARTH } \\
\hline$t$ & -519878 & -119425 & 0 & 0 \\
\hline & -518985 & -119630 & & \\
\hline \multirow[t]{2}{*}{$t^{2}$} & 136257 & -290016 & -105 & 236 \\
\hline & 135259 & -287427 & & \\
\hline$t^{3}$ & 101735 & 57019 & -402 & -305 \\
\hline$t^{4}$ & -17564 & 25773 & 336 & -160 \\
\hline$t^{5}$ & -6293 & -3225 & 196 & 243 \\
\hline$t^{6}$ & 1146 & -739 & -138 & 75 \\
\hline \multicolumn{5}{|c|}{ MARS } \\
\hline$t$ & 0 & 0 & 0 & 0 \\
\hline \multirow[t]{2}{*}{$t^{2}$} & -3584 & 22600 & 982 & -293 \\
\hline & -3599 & 22505 & & \\
\hline$t^{3}$ & -14815 & -3170 & -697 & 171 \\
\hline$t^{4}$ & 1616 & -5460 & -904 & -78 \\
\hline$t^{5}$ & 1346 & 89 & 243 & -299 \\
\hline$t^{6}$ & -108 & 226 & 267 & 85 \\
\hline
\end{tabular}

Table 3. Contribution of the Moon in the secular terms (Eq.8). The values in oldstyle are the corresponding values obtained by Bretagnon (1984), which include the second order terms due to the product of short period terms. The time $t$ is measured in units of 10000 julian years from J2000 (JD 2451 545.0). 


\section{References}

Abu El Ata, N., Chapront, J.: 1975, Astron. Astrophys. 38, 57- .

Berger, A., Imbrie, J., Hays, J., Kukla, G., Saltzman, B., eds. : 1984, Milankovitch and Climate, Reidel Publishing Company.

Bretagnon, P.: 1974, Astron. Astrophys. 30, 141-154.

Bretagnon, P.: 1982, Astron. Astrophys. 114, 278-288.

Bretagnon, P.: 1984a, Accuracy of long term planetary theory, in Milankovitch and Climate, Berger, A., et al. eds.

Bretagnon, P.: 1984b, Celest. Mech. 34, 193-201.

Bretagnon, p.: $1984 \mathrm{c}$, personal communication.

Bretagnon, P., Simon, J. L., Laskar, J. : 1985, Presentation of new Solar and Planetary Tables of interest for historical calculations. Journal for the History of Astronomy, submitted.

Brouwer, D., Van Woerkom, A. j.: 1950, APAE Vol. XIII, 2

Brumberg, V. A., Egorova, A. V.: 1971, Nablyudenya Iskustvenykh Nebesnykh Tel, 62, 42-72.(in russian)

Brumberg, V. A.: 1972, Relativistic Celestial Mechanics, Nauka, Moscow. (in russian)

Brumberg, V. A.: 1980, Analytical Algorithms of Celestial Mechanics, Nauka, Moscow. (in russian)

Chapront, J.: 1970,Astron. Astrophys. 7, 175- .

Chapront-Touzé, M., Chapront, J.: 1983,Astron. Astrophys. 124, 50-62.

Duriez, L.: 1977,Astron. Astrophys. 54, 93-112.

Duriez, L.: 1979, Approche d'une Théorie Générale Planétaire en variables elliptiques héliocentriques, Thèse, Lille.

Hill, G. W.: 1897, Astron. J. 17, 11

Kinoshita, H.: 1977, Theory of the rotation of the rigid Earth, Celest. Mech. 15, 277-326.

Laskar, J.: 1984, Théorie générale planétaire : éléments orbitaux des planètes sur un million d'années, Thèse de troisième cycle, Observatoire de Paris.

Laskar, J.: 1985a, Astron. Astrophys. 144, 133-146.

Laskar, J.: 1985b, Secular terms of classical planetary theories using the results of general theory, Astron. Astrophys. (submitted for publication).

Lestrade, J. F., Bretagnon, P.: 1982, Astron. Astrophys. 105, 42-52.

Newhall, X. X., Standish, E. M., Williams, J. G.: 1983, Astron. Astrophys. 125, 150-167. 